\title{
Index to Volume 2
}

\section{Articles}

Alary, Viviane [translated by Ann Miller], The Spanish Tebeo

Kunzle, David, The Gourary Töpffer Manuscript of Monsieur Jabot:

A Question of Authenticity. With the Dating and Distribution of

Rodolphe Töpffer's First Published Picture Story, and the World's First

Modern Comic Strip

Lefèvre, Pascal, The Conquest of Space: Evolution of Panel Arrangements and Page Layouts in Early Comics Published in Belgium (1880-1929)

Morgan, Harry, Graphic Shorthand: From Caricature to Narratology in

Twentieth-Century Bande dessinée and Comics

Plantu, 'I Must Not Draw ...'

Rossiter, Caroline, Early French Caricature (1795-1830) and English Influence

Vann, Michael G., Caricaturing 'The Colonial Good Life' in French Indochina

Weston, Jane, Bête et méchant: Politics, Editorial Cartoons and Bande dessinée in the French Satirical Newspaper Charlie hebdo

\section{Interviews}

Cabu and Tanitoc, Cabu Reporter [Interview of Cabu by Tanitoc]

\section{Reports}

Beaty, Bart, Angoulême, 2009

160

Beaty, Bart, Musée du Louvre, Paris, Le Petit dessein: Le Louvre s'ouvre au neuvième art, 22 January to 13 April 2009 


\section{Reviews}

Couvreur, Daniel and Frédéric Soumois, with a preface by Dominique Maricq, À la Recherche du Trésor de Rackham le Rouge (Raphaël Taylor)

Couvreur, Daniel, Frédéric Soumois and Philippe Goddin, Les Vrais Secrets de la Licorne (Raphaël Taylor)

Delisle, Philippe, Bande dessinée franco-belge et imaginaire colonial: Des Années 1930 aux années 1980 (Catriona MacLeod)

Fresnault-Deruelle, Pierre, Images à mi-mots (Ann Miller)

281

Hatfield, Charles, Alternative Comics: An Emerging Literature (Robert Duggan)

165

McLaughlin, Jeff, Comics as Philosophy (Catriona MacLeod)

162

Porret, Michel (ed.), Objectif bulles: Bande dessinée et histoire (Mark McKinney)

163

277 